\title{
Higiene y seguridad laboral de los trabajadores del vertedero municipal de la ciudad de Estelí en el II semestre 2015
}

\author{
Félix Sergio Talavera Rodríguez ${ }^{1}$ \\ Beverly Castillo Herrera²
}

\section{RESUMEN}

En el presente estudio investigativo se realizó una evaluación sobre. La Higiene y Seguridad Laboral de los Trabajadores del Vertedero Municipal de la Ciudad de Estelí en el II Semestre del 2015. Esta Investigación es de tipo Cuali - Cuantitativa (Mixta). Porque aborda a profundidad La Higiene y Seguridad Laboral de los trabajadores del Vertedero Municipal, Del Área de Servicios Municipales de la Alcaldía Municipal de la Ciudad de Estelí. Las principales Recomendaciones se enfocan en el seguimiento de Higiene y seguridad Laboral de los Trabajadores del Vertedero Municipal. El Universo de estudio lo constituyen: La Alcaldía Municipal de Estelí y su Área de servicios Municipales. Las Técnicas de Recolección de datos son: la Entrevista y Encuesta. Las entrevistas se realizaron al Ing. Carlos Pino Corrales Director del Área de Servicios Municipales y a Ing. Lelia Isabel Pérez Ruiz Jefe de Recolección y Tratamientos de Desechos sólidos. También se realizó Entrevista a trabajadores CPF. Y Churequeros. Se Realizó Encuesta a Trabajadores informales conocidos como Churequeros. Los principales resultados Reflejan la Situación de Higiene y Seguridad Laboral de los Trabajadores del Vertedero Municipal.

Palabras clave: Higiene y Seguridad Laboral.

Recibido: 25 de enero de 2016

Aceptado: 10 de junio de 2016

1 UNAN-Managua FAREM-Estelí, Correo Electrónico: felixtalarod@yahoo.es

2 Doctora en Ciencias Sociales por la Universidad del Zulia. Maestría en Ciencias Sociales por la Universidad de Guadalajara. Docente titular de la UNAN-Managua, FAREM-Estelí. Correo Electrónico: beverly.castillo@yahoo.com 


\title{
Hygiene and labor safety of the workers of the trash dump of Estelí, in the second semester of 2015
}

\begin{abstract}
The present study conducted an evaluation about hygiene and labor safety of the workers of the municipal trash dump of Estelí, in the second semester of 2015. This is a mix methods research (both quantitative and qualitative), as it deeply addresses the hygiene and safety of the workers of the municipal trash dump of Estelí. The universe of the study consisted of the Municipality of Estelí. The data collection instruments were interview and survey. The interview was administered to Carlos Pino Corrales, Director of the Municipal services, and to Lelia Isabel Perez, Director of Collection and Treatment of waste disposal. Interviews were also administered to security guards and land fillers. The main research results reveal that current situation related to hygiene and safety of the workers of the municipal trash dump.
\end{abstract}

Keywords: Hygiene, safety. 


\section{INTRODUCCIÓN}

Esta investigación se enfoca en la situación de higiene y seguridad laboral que viven los trabajadores formales e informales (churequeros) en el basurero de la ciudad de Estelí. A partir de la información se aplica un análisis de Fortalezas, Oportunidades, Debilidades y Amenazas (FODA) para proponer una estrategia que mejore las condiciones de los trabajadores y personas que llegan a depositar desechos en el basurero municipal.

El vertedero no tiene un mantenimiento adecuado para reducir los riesgos de enfermedades. Generalmente parte de la basura se quema, otra es movida por el viento, otra se la lleva el agua de lluvia, otra se profundiza en el suelo y hay basura que se convierte en vapor o gases tóxicos.

En este sitio existe todo tipo de contaminación producto de la basura proveniente de desechos humanos y químicos. La basura provoca gases tóxicos, humo, mal olor, roedores, y polvo. Así mismo, existen animales como roedores, moscas, mosquitos, zancudos y todo tipo de gérmenes que causan enfermedades. También existen zopilotes y perros que se bañan en los charcos de agua y se sacuden rociando agua en mal estado.

Todas las personas que están en el vertedero se ven perjudicadas en el sistema respiratorio y en el sistema digestivo. Así mismo, están expuestas a infectarse: piel, ojos, y pulmones. La basura es un gran problema para la salud de las personas, principalmente para los trabajadores que realizan la recolección en la ciudad y a quienes trabajan dentro del vertedero municipal.

Este estudio será de gran utilidad para la Alcaldía Municipal de Estelí porque sugiere estrategias para prevenir y reducir el problema de las enfermedades y los riesgos laborales en el vertedero municipal. Así mismo, esta investigación podrá ser de gran provecho para las instituciones u organismos interesados en aportar para la reducción de los riesgos de salud en las personas que diariamente llegan al vertedero municipal.
Los ejes teóricos más relevantes de la investigación son:

Gestión municipal, se define como la relación directa del estado con los habitantes de las distintas localidades del país, con los vecinos, con las personas que habitan con otras en una misma ciudad, pueblo, caserío o barrio (Pedrique, 2009).

Sistema de recolección de basura, conjunto de elementos que intervienen en la prestación o ejecución de tal servicio, donde podemos mencionar la forma en que se presta el servicio: público, privado, gratuito, con una tasa por la prestación, servicio amplio, integrado, reducido o Incompleto, específico (INIFOM \& AMUNIC, 2001)

Higiene y Seguridad Laboral, se entiende como seguridad ocupacional a las medidas o condiciones y seguridad del trabajo, que deben asegurar, dentro de todas las especies de servicios que se desarrollan en la vida, la salud y la integridad física de todo trabajador, relacionada estrictamente con la prevención de los accidentes laborales y enfermedades profesionales, su estudio corresponde en gran parte a la rama de seguridad social, dentro de las condiciones generales del trabajo, en que se deba desarrollar la actividad. (INATEC, 2002)

\section{MATERIALES Y MÉTODOS}

Esta investigación es de tipo cuali-cuantitativa (mixta) porque se analiza a profundidad la problemática de higiene y seguridad en el basurero municipal, y también se aplica un instrumento cuantitativo (encuesta) para analizar el comportamiento de las variables en los trabajadores formales y churequeros que laboran en esta Área de la Alcaldía Municipal.

El Universo de estudio es la Alcaldía Municipal de Estelí y su Área de servicios municipales. La muestra de estudio son los trabajadores que laboran en el vertedero municipal de la ciudad de Estelí, que son tres 
trabajadores formales y 40 trabajadores informales del vertedero municipal de Estelí. El tipo de muestreo es No probabilístico porque no todas las personas pueden ser seleccionadas. Los criterios de selección de la muestra para esta investigación han sido: Disposición de los informantes claves a ser entrevistados, participación voluntaria en el estudio, informantes claves en la institución con conocimientos y experiencia sobre la temática del estudio.

Se utilizaron la encuesta y la entrevista semiestructurada para obtener información. Se aplicaron 40 encuestas a trabajadores formales e informales del vertedero municipal y entrevistas. Así mismo, se aplicaron entrevistas a los siguientes funcionarios: Carlos Pino Corrales, Director de servicios municipales; Lelia Isabel Pérez Ruiz responsable de recolección de desechos sólidos y dos trabajadores CPF del vertedero municipal. Para procesar las encuestas se elaboraron tablas de frecuencia.

\section{RESULTADOS}

La alcaldía municipal de Estelí (AME), cuenta con una excelente estructura organizativa, conformada primeramente por el consejo municipal que lo integran los concejales de los diferentes partidos políticos, siendo la mayoría del partido gobernante. El consejo municipal, es el encargado de tomar las decisiones importantes y más relevantes para el desarrollo del municipio.

El siguiente nivel jerárquico es la Dirección General conformada por el Alcalde, vice -alcalde y el asesor legal. Bajo su responsabilidad están las distintas áreas de la alcaldía de Estelí como: Secretaria del medio ambiente, APROE, Adquisiciones, Divulgación y prensa y Secretaria de la mujer. El siguiente nivel son los Departamentos: Dirección financiera, Servicios municipales, Obras municipales, Planificación, y Participación ciudadana.
El área de Servicios municipales de la Alcaldía municipal de Estelí, es la encargada de prestar los servicios básicos a toda la población, realizar mantenimiento a toda la maquinaria que se utiliza en la prestación de los servicios y la organización de todo el personal que labora para determinada área, tratando siempre de brindar un buen servicio que permita el bienestar de la población.

\section{La organización del sistema de ruteo para la recolección en la ciudad de Estelí}

En la organización del sistema de ruteo para la recolección de basura es el área de mayor demanda de servicios municipales, está diseñada en rutas de recolección. Se comienza a recolectar la basura desde las $6.00 \mathrm{am}$. Tiene una cobertura del $96 \%$ en base a barrios de la ciudad.

Tiene macro ruteo y micro ruteo. Para estas solo hay personas encargado y fiscal. Existen 5 rutas de recolección de basura y una ruta especial. De las cuales 5 son rutas domiciliares y se visitan a diario y una ruta especial que se visita una vez a la semana. Estas rutas son equipadas con un camión recolector de basura con sus ayudantes. El área de servicios municipales cuenta con siete camiones de basura y un tráiler para la recolección de los desechos debidamente equipados. Los trabajadores del área de recolección de basura que llegan a botar basura gozan de los derechos de protección. Los equipos consisten en: uniformes, botas, mascarilla, y guantes.

El costo del servicio de recolección corresponde al tributo que se paga de 24 córdobas (Equivalente a un dólar americano al mes). Se le suma el pago de limpieza de calles que es de $\mathrm{C} \$ 15.00$ (córdobas para los barrios. Para los grandes negocios como: pulperías, multicentro, gasolineras, tiendas, etc., el pago es mayor.

Situación de higiene y seguridad laboral en el vertedero municipal 
En el vertedero municipal no se cuenta con una organización establecida y de gran tamaño. En el vertedero municipal laboran dos vigilantes $(\mathrm{CPF})$, el operador del tractor que atierra la basura y los choferes y ayudantes que manejan los Camiones recolectores de basura que llegan a depositar la basura al vertedero. El resto son Churequeros, que no tienen ninguna protección laboral. La Alcaldía le da seguimiento al trabajo del vertedero a través del Área de Servicios Municipales.

Los trabajadores formales del vertedero municipal no cuentan con una protección adecuada a sus funciones, siendo un trabajo de alta peligrosidad de sufrir accidentes como de contraer enfermedades que pueden afectar seriamente su salud.

La mayoría de la población que se beneficia del servicio de recolección de basura no realiza el pago en tiempo y forma, lo que ocasiona pocos ingresos a la comuna; $\mathrm{y}$ esto repercute en la liquidez necesaria para equipar adecuadamente al personal.

Se procesaron cuarenta encuestas que permitieron obtener un análisis más detallado sobre la situación de higiene y seguridad laboral de los trabajadores informales del vertedero municipal de la ciudad de Estelí. La mayoría de los trabajadores informales no cuentan con medidas de seguridad; por lo tanto se encuentran expuestos a contraer enfermedades.

\section{Datos generales de los trabajadores informales encuestados}

Como dato general en las encuestas, se obtuvo la edad de los trabajadores informales, el 40\% de los trabajadores tiene una edad de 20-30 años, el 28\% presentan una edad 10-20 años, el 18\% de los churequeros tienen una edad 30-40 años, el 14\% tiene de 40 años a más. Estos datos muestran que existe bastante presencia de gente joven -entre 20-30 años- en el vertedero, lo cual debe de ser motivo de preocupación para la población y las distintas autoridades de la ciudad.
Un $55 \%$ de los encuestados son del sexo masculino y el $45 \%$ del sexo femenino. El 33\% son solteros porque la mayoría son personas jóvenes; el $30 \%$ tienen una relación estable; y un $27 \%$ de los trabajadores informales son divorciados/solteros. Apenas un $4 \%$ son casados. Las personas que afirmaron ser divorciadas o separadas tienen hijos y deberían de tener un trabajo formal para satisfacer sus necesidades y criar mejor a sus hijos e hijas. El 81\% de los encuestados tienen hijas mujeres y el 59\%, tienen hijos varones. Solamente dos personas (9\%) afirmó no tener hijos

\section{El trabajo en el vertedero municipal de los trabajadores informales}

El 75\% de las personas afirman que les gusta realizar las labores en el vertedero municipal y solo el $25 \%$, respondieron que no les gusta su trabajo.

"Trabajo en el Vertedero Municipal porque estoy desempleado es un trabajo regular hay que tomar en cuenta que la necesidad los obliga a estar aquí en este lugar recogiendo basura" (Entrevista realizada al churequero Juan. 13 noviembre 2015).

Mientras un 73\% de los trabajadores informales realiza labores por necesidad de sobrevivir. Mientras el 18\% trabaja porque está desempleado; 3 (8\%) trabajadores realizan labores para obtener ingresos y un churequero $(2 \%)$ dijo que trabaja porque le gusta y no tienen preparación.

"Quienes realizan la labor de churequeros en el vertedero municipal es porque tienen necesidad no es por gusto". (Entrevista realizada al churequero Alfredo el 13 de noviembre del 2015).

El $72 \%$ de los trabajadores informales del vertedero municipal valoran su trabajo como regular; el 18\% (7 churequeros) opinan que el trabajo en es malo y el $10 \%$ (4 trabajadores) refieren que es bueno. 
Los beneficios que se tiene de trabajar en el vertedero, el $58 \%$ de los trabajadores (23) realizan las labores en el vertedero porque los ingresos obtenidos de la basura les permite satisfacer sus necesidades; 12 churequeros $(30 \%)$, realizan labores por desempleo, nadie los contrata, no tienen preparación mínima o estudios que requieran para un trabajo formal, y logran un ingreso diario para cubrir sus necesidades básicas. El 10\% (4 trabajadores) consideran que tienen un trabajo; y solamente el 2\% (1 trabajador) realizan labores porque tienen un trabajo fijo y otro $2 \%$ porque se relacionan con otros trabajadores en el basurero.

"Yo tengo bastante tiempo de recoger chatarra y gracias a Dios logro satisfacer mis necesidades. Pero sería bueno que nos brinden trabajo más saludable o alguna ayuda". (Entrevista a Angelita. Churequera del vertedero. 13 de noviembre 2015).

Los trabajadores informales que trabajan en el vertedero municipal, los productos que recolectan mayoritariamente son: aluminio (93\%), latas (58\%) y el $38 \%$ recolecta otros materiales como: cobre, bronce, entre otros utensilios que son reutilizables por diferentes personas o por ellos mismos. El canje o la venta de estos materiales les permiten obtener productos básicos para su sobrevivencia.

El 30\% realiza recolecta de botellas que son vendidas para ser reutilizadas y presentan bastante demanda entre los clientes; y un 32\% recolecta cartón para la comercialización.

"Los materiales que más se obtienen del vertedero son: Aluminio, Latas, botellas, cartón y otros entre ellos hierro, cobré bronce”. (Entrevista a Luis. Churequerodelvertedero.13 denoviembrede12015).

Los productos que mejor paga reciben los churequeros por orden de importancia son: el Aluminio con un 55\%; el hierro (40\%) y el Cobre con un 5\%. El cobre la libra cuesta más que el aluminio y el hierro; pero es más escaso de encontrar y entre el reciclaje y su obtención, requiere de más procesos.
"Cuando vienen a comprar materiales los precios en córdobas son los siguientes: papel un quintal = 100:00 córdobas; cartón un quintal $=50: 00$ córdobas; aluminio un quintal $=800: 00$ córdobas, cobre una libra $=35: 00$ córdobas, botellas de vidrio el cien $=35: 00$ córdobas, botellas de plástico un quintal = 100:00 córdobas, hierro un quintal $=$ 80:00 córdobas". (Entrevista a Francisca. Churequera. 13 de noviembre del 2015).

El $50 \%$ de los churequeros afirma que obtiene un ingreso promedio de C\$500-C\$1000 al mes. Mientras el 25\% obtienen ingresos de C\$1500-C\$2000; el 3\% obtiene ingresos de C\$1000-C\$1500 y el 2\% obtiene ingresos de C\$100-C\$500.

"Los churequeros vienen al vertedero a ejercer su labor por necesidad la que no todos satisfacen debido al poco ingreso que obtienen no llegan al salario mínimo. Al compararlos con los trabajadores formales del vertedero o de los recolectores de basura estos ganan más del salario mínimo,tienen prestaciones sociales, hacen horas extras, reciben bono solidario y bono mensual, algunos reciben viáticos, gozan de equipos de protección, tienen INSS, derecho a clínica”. (Ing. José Ángel Pino. Responsable de Servicios Municipales de la Alcaldía de Estelí).

\section{Riesgos laborales y enfermedades en el vertedero municipal}

En el cuadro No.23, se observa que un $48 \%$ de los churequeros, es decir 19 trabajadores informales, respondieron que los principales padecimientos que presentan son dolores en la espalda y huesos; un $40 \%$ tienen enfermedades respiratorias; el $27 \%$ enfermedades de la piel como alergias y el $20 \%$ enfermedades por intoxicación por humo y químicos.

"Aqui las enfermedades que más padecen los churequeros son: enfermedades de la piel, dolores de espaldas y huesos, calentura, tos y alergias" 
(Entrevista a Armando. Churequero. 13 de noviembre del 2015).

Las dos principales enfermedades que padecen los trabajadores informales del vertedero municipal, el 65\% dijo que era dolor de cabeza, un $40 \%$ las enfermedades respiratorias presentan y un $20 \%$ respondieron otras enfermedades como: alergia, dolor en la espalda, manchas en la piel, dolores musculares, otras.

Los trabajadores informales del vertedero municipal se enferman periódicamente, el $45 \%$ le sucede una vez a la semana y otro $40 \%$ afirma que se enferma una vez al mes. En menor porcentaje están quienes se enferman cada tres meses ( $8 \%)$, una vez al año $(2 \%)$ y dos veces al año el $2 \%$. Cabe destacar que ninguno de los encuestados afirma que no se ha enfermado desde que trabaja en el vertedero municipal.

Los churequeros respondieron que cuando se enferman asisten al centro de salud (52\%); 3 trabajadores (7\%) asisten al hospital y 18 trabajadores (45\%) se auto medican.

"Cuando se enferman acuden al centro de salud, Compran una pastilla, van al hospital". (Entrevista a José. Churequero. 13 de noviembre del 2015).

En relación a los riesgos laborales por trabajar en el vertedero, el $67 \%$ afirma que son caídas o resbalones, un $27 \%$ las cortaduras, el $20 \%$ insertarse objetos y el $15 \%$ las quemaduras. Mientras un 5\% se refiere a otros riesgos como: torceduras, e intoxicación. Cabe destacar que apenas el 2\% (un trabajador) dijo que tiene en el vertedero no había riesgo laboral.

En los tipos de accidentes que han sufrido los trabajadores informales, están: un $62 \%$ caídas y resbalones, el 20\% cortaduras; y un 10\% golpes. En menor porcentaje están un 5\% que han sufrido quemaduras (2 trabajadores) y un $7 \%$ que se han insertado objetos. Cabe señalar que un 17\% afirma no haber sufrido ningún accidente laboral.
"He tenido accidentes como son: caídas, cortaduras con vidrios". (Entrevista a Juana. Churequera. 13 de noviembre del 2015.).

Los churequeros cuando sufren un accidente laboral, el $60 \%$ va al centro de salud, el $35 \%$ se auto medican y si es grave, un $12 \%$ acude al hospital.

"Al ocurrir un accidente laboral acuden al centro de salud, en ocasiones se curan ellos mismos, ejemplo si se cortan con vidrio se amaran un trapo y se toman pastilla”. (Entrevista realizada a Carmela. Churequera. 13 de noviembre del 2015).

\section{Higiene y seguridad en el vertedero municipal}

En higiene y seguridad de los informales del vertedero municipal, sobre las medidas de higiene y seguridad laboral que utilizan que utilizan, el 97\% respondieron que no utilizan medidas de seguridad y apenas un 3\% afirman utilizar medidas de seguridad.

Mientras el 52\% (21 trabajadores) no utilizan ninguna media de seguridad. El $27 \%$ sólo usa pañuelos, otro $15 \%$ usa botas, el $10 \%$ gorras o sombreros para protegerse del sol y el 3\% usan ropas especiales y guantes. Se destaca el $52 \%$ que afirma no utilizar ninguna medida de protección.

"Para protección solo utilizamos un pañuelo para colocárselo en la nariz. No tenemos recursos para conseguir equipos de protección”. (Entrevista a Mario. Churequero 13 de noviembre del 2015).

E1 79\% de los encuestados respondieron que no utilizan medidas de seguridad porque no tienen recursos, ni materiales. Apenas un trabajador (3\%) afirmó que utiliza medidas de seguridad.

El 97\% de los encuestados aseveró que nunca han recibido supervisión del Ministerio del Trabajo (MITRAB) para valorar las condiciones de trabajo y 
su situación de salud. Sólo un trabajador (3\%) afirmó lo contrario.

"No hemos recibido visita del ministerio del trabajo, aqui se han olvidado de nosotros". (Entrevista a Juan de la chica.Churequero. 13 de noviembre del 2015).

Sin embargo, hay otros organismos que visitan el vertedero, el 12\% (5 trabajadores) atestiguo que han llegado grupos evangélicos y el $7 \%$ han recibido visitas de personas particulares.

"Vienen visitas de grupos evangélicos al vertedero municipal pocas veces nos brindan comida o refrigerio a cambio de escuchar el culto que ellos realizan”. (Entrevista a Maricela. Churequera. Noviembre 2015).

En lo referente al tipo de ayuda que reciben los trabajadores del vertedero, el 17\% afirmó que les han regalado alimentos, al $12 \%$ ropa y zapatos y otro $12 \%$ respondió que han recibido cosas de higiene personal, plástico para sus casas u otro objeto que ya no es de utilidad para los organismos y sólo un trabajador (2\%) ha recibido medicina. Destaca el $65 \%$ que afirmó no recibir ningún tipo de ayuda.

"Se ha recibido ayuda en poca cantidad de alimento, ropa, zapatos, frescos por parte de algunos grupos de evangélicos y personas particulares". (Entrevista a Carelia.Churequera.13 de noviembre del 2015).

\section{Análisis de Fortalezas, Oportunidades, Debilidades y Amenazas (FODA) del vertedero municipal}

\section{Fortalezas}

- Está ubicado en un lugar Adecuado para vertedero

- Personal capacitado para el desempeño de sus funciones

- Fuente de generación de ingresos para los churequeros

\section{Debilidades}

- Contaminación ambiental.

- Ya dio su vida útil como vertedero

- Exposición a enfermedades.

- Vulnerable a riesgos y accidentes.

- No presenta condiciones para relleno sanitario.

- Desorganización de los churequeros.

- Poca visita de organismos e instituciones.

- Pocos vehículos recolectores de desechos solidos

- Exposición a accidentes, cortaduras, quemaduras y enfermedades comunes

\section{Oportunidades}

- Realizan labores beneficiosas para los trabajadores de la institución y churequeros.

- Obtención de productos reciclables que generen ingresos a los churequeros.

- Realización de estudios para diferentes instituciones. 
Propuesta de estrategia para la mejora de higiene y seguridad en el vertedero del municipio de Estelí.

Línea estratégica 1: Mejora de condiciones de Higiene y Seguridad

Objetivo general: Reducir la contaminación ambiental y las enfermedades en los trabajadores formales del vertedero municipal.

Línea estratégica 2: Reorganización del vertedero.

Objetivo general: Incrementar la vida útil mediante el aprovechamiento óptimo del espacio del vertedero municipal

Línea estratégica 3: Equipar con equipo y maquinaria la recolección y transporte de desechos sólidos.

Objetivo general: Garantizar el buen servicio a la población mediante una eficaz recolección de basura en la ciudad de Estelí.

Línea estratégica 4: Estudio ambiental del basurero municipal

Objetivo general: Garantizar la salud de la población obteniendo un ambiente saludable.

Línea estratégica 5: Atención a los trabajadores informales (Churequeros)

Objetivo general: Valorar la situación actual de los trabajadores informales del vertedero municipal para la coordinación de acciones con instituciones $\mathrm{u}$ organismos que ayuden a mejorar su situación actual

\section{CONCLUSIONES}

El proceso de recolección de desechos sólidos del vertedero municipal de la ciudad de Estelí, está equipado con siete camiones recolectores y 1 tráiler que se encargan de recolectar los desechos sólidos de la ciudad de Estelí, distribuidos u organizados por rutas y barrios, especificados en los cuadros.

Sobre la situación de higiene y seguridad laboral en el vertedero municipal de los churequeros u otras personas, el $97 \%$ no utilizan medidas de higiene y seguridad laboral porque no tienen y solamente el 3\% utiliza medidas de higiene y seguridad laboral.

Se sugiere priorizar la línea estratégica No.1 referida a la mejora en las condiciones de higiene y seguridad laboral de los trabajadores formales que desempeñan labores en el vertedero municipal.

\section{BIBLIOGRAFÍA CITADA}

Instituto Nacional Tecnológico. (2002). Medidas de higiene y seguridad ocupacional. Manual para el participante Medidas de higiene y seguridad del trabajo. Managua: INATEC. Obtenido de http:// www.mific.gob.ni/Portals/0/Documentos\%20 Fomento/PROYECTOS\%20FOMENTO/ P R A M E C L IN / M A N U A L \% 20 D E \% 20 MEDIDAS\%20DE\%20HIGIENE\%20Y\%20 SEGURIDAD\%20OCUPACIONAL.pdf

Instituto Nicaragüense de Fomento Municipal (INIFOM) \& Asociación de Municipios de Nicaragua (AMUNIC). (2001). Manual elemental de Recolección y tratamiento de Desechos Sólidos. Managua: INIFOM. Obtenido de http://www.inifom.gob.ni/areas/Documentos/ Instrumentos $\% 20 \mathrm{SSMM} / \mathrm{manua} 1 \% 20 \mathrm{de} \% 20$ desechos $\% 20$ s\%C3\%B3lidos.pdf

Pedrique, J. (2009). SlideShare. Obtenido de SlideShare: http://es.slideshare.net/jcupertino/ gestin-municipal-1488285 